\title{
Average consensus in asymmetric broadcasting wireless sensor networks through gossiping
}

\author{
Ferdinand Peper \\ Center for Information and Neural Networks \\ NICT and Osaka University \\ Osaka, Japan \\ peper@nict.go.jp \\ Tetsuya Shimokawa \\ Center for Information and Neural Networks \\ NICT and Osaka University \\ Osaka, Japan \\ shimokawa@nict.go.jp
}

\author{
Kenji Leibnitz \\ Center for Information and Neural Networks \\ NICT and Osaka University \\ Osaka, Japan \\ leibnitz@nict.go.jp \\ Marie-Ange Remiche \\ Faculty of Computer Science \\ University of Namur \\ Namur, Belgium \\ Marie-Ange.Remiche@unamur.be
}

\begin{abstract}
Average consensus algorithms have attracted increasing interest in the last decade because of their potential for use in high-density wireless sensor networks. This paper analyzes an algorithm that is based on a model of asymmetric broadcasting on a random geometric graph, in which nodes broadcast and listen only intermittently. We show that each node can easily estimate its update weight from the degree of silence in its neighborhood, and, after the weights have been assigned in this way, that the consensus algorithm converges to the true average. Both a synchronous and an asynchronous update model are analyzed.
\end{abstract}

\section{CCS Concepts}

- Computing methodologies $\rightarrow$ Self-organization;

- Human-centered computing $\rightarrow$ Mobile computing;

\section{Keywords}

Average consensus; gossiping algorithms; wireless sensor networks

\section{INTRODUCTION}

In the coming decade significant growth is expected in mobile traffic to support human-centric and machine-type interactions. The Internet of Things (IoT) is one driving

\footnotetext{
* Corresponding author

Permission to make digital or hard copies of all or part of this work for personal or classroom use is granted without fee provided that copies are not made or distributed for profit or commercial advantage and that copies bear this notice and the full citation on the first page. Copyrights for components of this work owned by others than the author(s) must be honored. Abstracting with credit is permitted. To copy otherwise, or republish, to post on servers or to redistribute to lists, requires prior specific permission and/or a fee. Request permissions from permissions@ acm.org.

MOBIQUITOUS '16 Adjunct Proceedings, November 28-December 01, 2016, Hiroshima, Japan

(C) 2016 Copyright held by the owner/author(s). Publication rights licensed to ACM. ISBN 978-1-4503-4759-4/16/11 ..\$15.00

DOI: http://dx.doi.org/10.1145/3004010.3004024
}

force behind this growth, with the number of IoT devices expected to grow from more than 15 billion today to 50 billion in 2020 [8]. The sheer amount of wireless traffic resulting from this will be overwhelming and difficult to realize with the conventional technology now available, due to interference between devices and network congestion. This requires a rethinking of the way information is processed in ultra-dense small sensor networks [9], which have started to attract increasing attention recently. The huge number of nodes required in such networks will make central control mechanisms infeasible, and they also run into the limitations on wireless resources. It will be difficult to supply all devices in such networks with energy for extended times, because individually charging their batteries will be impractical due to their large numbers, so there will be a heavy reliance on energy harvested from the environment. The opportunistic nature of energy harvesting, however, will affect the reliability of network operation. Since the operating constraints of this type of networks are very different from conventional technology, a fundamental reconsideration of designs is necessary.

In many cases the information required from these networks will be limited to statistics measured over a large number of nodes, such as the maximum of the temperatures of perishable products, or their average, etc. The typical mode of operation in conventional sensor networks, in which the individual measurements of all nodes are routed to a central server, is thus no longer necessary in practice; rather, the server can receive the essential information from a small set of nodes in the network. It thus pays off to design algorithms that extract statistical information in a local, distributed, way. These algorithms should be able to operate in a highly unreliable environment, in which collisions between wireless messages and their drop-out occurs with a non-zero probability, and nodes will not even know whether and which other nodes have received their broadcasts.

Consensus algorithms $[12,13,10]$ are distributed algorithms in which nodes reach a consensus on a value in the network that is a function of the values of all individual 
nodes. This value may for example be the average, the maximum or minimum, and the majority value from a finite set of values. Especially when used with a gossiping mode of interaction [5], which means that each node only communicates with nodes in its local neighborhood, consensus algorithms are very suitable for the framework outlined above.

This paper investigates an average consensus algorithm based on gossiping on a random geometric graph $[7,11$, $2,3]$ in which each node broadcasts its value with a given probability in an asymmetric way, i.e., it will not receive acknowledgments from nodes that have received its value, and neither does it even know the IDs of the nodes that can receive its broadcasts. On the receiving side, each node has a given probability of listening to broadcasts from its neighbors, without being aware where those neighbors are and without knowing their IDs. We show that the algorithm converges to the average of the node values. The model includes the possibility that broadcasts of nodes collide, which will be ignored at the receiver's side, and drop-out of messages are assumed to occur with a certain probability.

This paper is organized as follows. In Section 2 we describe the model for the case all nodes are updated in lockstep with each other (synchronous updating). Section 3 analyzes an asynchronous model in which nodes broadcast or listen according to a marked Poisson process. Section 4 shows the numerical evaluation of the asynchronously updated model. We finish this paper with conclusions in Section 5 .

\section{SYNCHRONOUS UPDATING}

Assume $G=(V, A)$ is an undirected graph, whereby $V$ is a finite set of $n=|V|$ nodes and $A$ is the $(0,1)$-adjacency matrix of the graph, with $a_{i i}=0$, i.e., the graph has no selfloops. The neighborhood $N_{i}$ of node $i$ consists of all nodes $j$ for which $a_{i j}=1$. Since $G$ is undirected, the adjacency matrix is symmetric.

In synchronous updating, time is divided into equal intervals and all nodes are active at the same iteration. The action undertaken by each node is the update of its value, or, alternatively, the decision not to update in the current iteration.

Define a boolean condition $B(i, k)$ that denotes whether node $i$ broadcasts at iteration $k$, and assume that the probability $p_{b}=\operatorname{Pr}[B(i, k)]$ of this happening is constant (not dependent on node or iteration). We further define a boolean condition $L(i, k)$ that denotes whether node $i$ listens to any broadcasts from its neighbors at iteration $k$, and we make a similar assumption for the corresponding probability $p_{l}=$ $\operatorname{Pr}[L(i, k)] . \quad B(i, k)$ and $L(i, k)$ are mutually exclusive conditions, so a node cannot broadcast and listen at the same time. The probability that a node is inactive (sleeping) is $p_{s}=1-p_{b}-p_{l}$. We assume that a node can detect when more than one of its neighbors is broadcasting in the same iteration. In that case there is a collision, and the node will not accept any broadcast in that iteration.

Let $x_{i}(k)$ be the value of node $i$ at iteration $k$. The average consensus algorithm according to which nodes are updated is as follows:

$$
x_{i}(k)= \begin{cases}x_{i}(k-1)+\frac{\alpha}{\omega_{i}}\left[x_{j}(k-1)-x_{i}(k-1)\right] \\ \text { if } C(i, j, k-1) \\ x_{i}(k-1) & \text { otherwise }\end{cases}
$$

where $C(i, j, k) \equiv L(i, k) \wedge B(j, k) \wedge \forall r \in N_{i} \backslash\{j\}: \neg B(r, k)$, and $\alpha$ is the update rate and $\omega_{i}$ is a weight that is set such that on average the value by which node $i$ is updated in an iteration is the same as that for any other node $j$. The parameters $\alpha$ and $\omega_{i}$ satisfy the inequality $0<\alpha / \omega_{i}<1$. According to Eq. (1), node $i$ is updated if exactly one of its neighbors is broadcasting and it itself is listening. Node $i$ has only access to the value of the neighboring node that broadcasts, but it is unaware of that neighbor's identity.

In order to analyze the convergence behavior of the update algorithm, we average it over all possible broadcasting and listening scenarios. Let $m_{i}(k)=\mathrm{E}\left[x_{i}(k)\right]$ be the expectation of the value of node $i$. Then,

$$
\begin{aligned}
m_{i}(k)= & \sum_{j \in N_{i}} \operatorname{Pr}[C(i, j, k-1)] \cdot \\
& \left\{m_{i}(k-1)+\frac{\alpha}{\omega_{i}}\left[m_{j}(k-1)-m_{i}(k-1)\right]\right\} \\
& +\left(1-\sum_{j \in N_{i}} \operatorname{Pr}[C(i, j, k-1)]\right) m_{i}(k-1) \\
= & \left(1-\frac{\alpha}{\omega_{i}}\left|N_{i}\right| p_{l} p_{b}\left(1-p_{b}\right)^{\left|N_{i}\right|-1}\right) m_{i}(k-1)+ \\
& \frac{\alpha}{\omega_{i}} p_{l} p_{b}\left(1-p_{b}\right)^{\left|N_{i}\right|-1} \sum_{j \in N_{i}} m_{j}(k-1)
\end{aligned}
$$

In matrix form we then obtain

$$
\mathbf{m}(k)=S \mathbf{m}(k-1),
$$

whereby $\mathbf{m}(k)=\left(m_{1}(k), \ldots, m_{n}(k)\right)^{T}$, and the matrix $S$ has as its elements

$$
s_{i i}=1-\frac{\alpha}{\omega_{i}}\left|N_{i}\right| p_{l} p_{b}\left(1-p_{b}\right)^{\left|N_{i}\right|-1}
$$

in the diagonal,

$$
s_{i j}=\frac{\alpha}{\omega_{i}} p_{l} p_{b}\left(1-p_{b}\right)^{\left|N_{i}\right|-1}
$$

outside of the diagonal $(i \neq j)$ for $j \in N_{i}$, and

$$
s_{i j}=0
$$

otherwise $\left(i \neq j\right.$ and $\left.j \notin N_{i}\right)$. An element $s_{i j}$ is zero if and only if $a_{i j}$ is zero. Moreover, $S$ is a stochastic matrix, because $\sum_{j} s_{i j}=1$ for all $i$, which implies that the average consensus of all nodes is a fixed point of equation (2).

In order to guarantee that the sum of all nodes is preserved on average in the update process it is necessary in addition that $S$ is a doubly stochastic matrix [12], i.e., $\sum_{i} s_{i j}=1$ for all $j$. This requires that the inner product of the 1 -vector and column $i$ of matrix $S$ equals one, i.e.,

$\mathbf{1}^{T} s_{\bullet i}=1-\alpha p_{l} p_{b} \sum_{j \in N_{i}}\left[\frac{\left(1-p_{b}\right)^{\left|N_{i}\right|-1}}{\omega_{i}}-\frac{\left(1-p_{b}\right)^{\left|N_{j}\right|-1}}{\omega_{j}}\right]=1$

We thus need to find values of $\omega_{i}$ and $\omega_{j}$ such that for all pairs $i$ and $j$ the following holds.

$$
\frac{\omega_{j}}{\omega_{i}}=\frac{\left(1-p_{b}\right)^{\left|N_{j}\right|-1}}{\left(1-p_{b}\right)^{\left|N_{i}\right|-1}}
$$

Since node $i$ has no information available about other nodes, the best that can be done to satisfy this equality is to require that $\left(1-p_{b}\right)^{\left|N_{i}\right|-1} / \omega_{i}$ is constant. This means that $\omega_{i}$ has the form

$$
\omega_{i}=c\left(1-p_{b}\right)^{\left|N_{i}\right|-1}
$$


with $c$ being a constant that does not depend on node $i$. Now, the quantity $\left(1-p_{b}\right)^{\left|N_{i}\right|}$ is easy to estimate, because it equals the probability that all nodes in the neighborhood of node $i$ are silent (not broadcasting) in iteration $k$. Unlike in [6], it is not necessary to know or estimate the values of $p_{b}$ and $\left|N_{i}\right|$ individually. The resulting average consensus algorithm then consists of two stages, i.e., a stage in which the proper weights are assigned to nodes through estimation of the probability of a silent neighborhood, followed by a stage in which average consensus is reached. An estimation algorithm for $\omega_{i}$ when $c=1-p_{b}$ is for example:

$$
\omega_{i}(k)=\omega_{i}(k-1)+\frac{1}{k}\left(y_{i}(k)-\omega_{i}(k-1)\right),
$$

whereby $y_{i}(k)$ is a Bernoulli distributed r.v. that is 1 if no broadcast is detected in the neighborhood of node $i$ at iteration $k$ and 0 otherwise.

If the $\omega$ 's are balanced according to Eq. (3), the system in Eq. (2) will converge to average consensus according to Theorem 2 in [10], provided that the following condition is satisfied.

$$
0<\alpha<\min _{i=1 \ldots n} \frac{\omega_{i}}{\left|N_{i}\right| p_{l} p_{b}\left(1-p_{b}\right)^{\left|N_{i}\right|-1}}
$$

Substituting the $\omega$ 's in Eq. (3) to balance for unequal node degrees gives us the following condition:

$$
0<\alpha<\min _{i=1 \ldots n} \frac{c}{\left|N_{i}\right| p_{l} p_{b}}
$$

whereby $c$ is a constant determined such that the $\omega$ 's are all within a range that optimizes convergence speeds, while guaranteeing stable dynamics of Eq. (1).

The convergence speed is determined by the second largest eigenvalue $\mu_{2}$ of matrix $S$ [10], while the largest eigenvalue has no such influence, since it always equals 1 . The smaller the value of $\mu_{2}$ the faster the convergence towards average consensus will be, and in order to obtain a sufficiently low value of $\mu_{2}$ we need, as a rule of thumb, the non-zero offdiagonal elements of matrix $B$ to be as large as possible, because this will bias the diagonal elements away from the value 1. Substituting expression (3) for $\omega_{i}$ into $s_{i j}$, we obtain

$$
s_{i j}=\alpha p_{l} p_{b} / c \text {. }
$$

Eq. (5) then implies

$$
s_{i j}<\frac{1}{\max _{r}\left|N_{r}\right|}
$$

for $i \neq j$. So, faster convergence will be achieved when the off-diagonal elements of $S$ are as close as possible to the reciprocal of the graph's maximum node degree but not exceeding it.

\section{ASYNCHRONOUS UPDATING}

When each node has its own schedule according to which it is activated we call the update scheme asynchronous. Each node in an asynchronous update scheme has its own clock, which runs independently of the clocks of other nodes. In the literature $[1,4]$ asynchronous updating has been modeled by using a Poisson process for each node with rate $\lambda$. For a total of $n$ nodes the superposition of these processes is equivalent to a Poisson process with rate $n \lambda$. When the times of the events in this process are denoted by $Z_{k}$, the time line is divided in intervals $\left[Z_{k}, Z_{k+1}\right)$ with exponentially distributed length, whereby $k$ is an index parameter that assumes a discrete value. Updates are then done one node at a time in a way as if the intervals $\left[Z_{k}, Z_{k+1}\right)$ are regular clock intervals in a synchronous scheme.

There are several problems with this approach. First, the lengths of the subsequent intervals are dependent on the number of nodes in the network: the more nodes the shorter each interval will be on average. Since each node requires a certain minimal time period to conduct a broadcast or to listen to broadcasts from other nodes, the model will not reflect reality beyond a certain number of nodes. Second, the probabilities of broadcast in an interval are not necessarily constant, but depend on the length of the interval.

To address this issue, we will assume that each node has constant-length time intervals $T_{b}$ respectively $T_{l}$ for broadcasting and listening $\left(T_{b}<T_{l}\right)$, which are initiated by events that are described as marked Poisson processes with a probability $p_{b}$ that an event initiates a broadcast, and probability $p_{l}=1-p_{b}$ that the event initiates listening. When a node is not broadcasting or listening we consider it asleep. A sleep state is not initiated by an event, however. Rather, it is the default state in between listening or broadcast intervals of a node. There is no minimum time interval between two successive Poisson events from the same node, so it is possible that a node's interval for broadcast or listening is interrupted by an event of its own, but this is addressed in the model by not conducting an update in that iteration. Eventually that node will have a broadcast or listening interval that is not interrupted by an event of its own, and this interval will then define the iteration in which the action of the node takes place. The update equation used in the asynchronous case is the same as in the synchronous case, i.e., we use (1), but we redefine the boolean condition for $C(i, j, k)$ and assume that each node uses its own timer to track its index $k$. To this end, we first define boolean conditions that are used in the definition of $C(i, j, k)$. This condition is defined in terms of three other conditions, which are listed below.

\begin{tabular}{|l|l|}
\hline Condition & Definition \\
\hline$L(i, k)$ & $\begin{array}{l}\text { Node } i \text { is in the listening state at its } \\
\text { iteration } k \text { and no other (broadcast } \\
\text { or listening) events of its own will } \\
\text { occur during its listening interval. }\end{array}$ \\
\hline$I(i, j, k)$ & $\begin{array}{l}\text { One broadcast interval of node } j \\
\text { falls inside a listening interval of } \\
\text { node } i \text { at iteration } k \text { of node } i .\end{array}$ \\
\hline$O(i, j, k)$ & $\begin{array}{l}\text { One or more broadcast intervals } \\
\text { from node } j \text { overlap the listening } \\
\text { interval of node } i \text { at iteration } k \text { of } \\
\text { node } i .\end{array}$ \\
\hline
\end{tabular}

Figs. 1 and 2 illustrate when conditions $I$ resp. $O$ are satisfied. Condition $C(i, j, k)$ is now defined as follows:

$$
C(i, j, k) \equiv L(i, k) \wedge I(i, j, k) \wedge \forall r \in N_{i} \backslash\{j\}: \neg O(i, r, k)
$$

In other words, condition $C(i, j, k)$ is satisfied iff node $i$ is listening at its iteration $k$ and is not interrupted in doing so by other events of its own, and node $j$ is broadcasting while its broadcast interval falls inside the listening interval of node $i$, and no other nodes in the neighborhood of node $i$ broadcast while their broadcast interval overlaps the listening interval of node $i$.

In order to translate update equation Eq. (1) under the new condition $C(i, j, k)$ into an averaged equation, we determine the probabilities of the partial conditions in $C(i, j, k)$. 

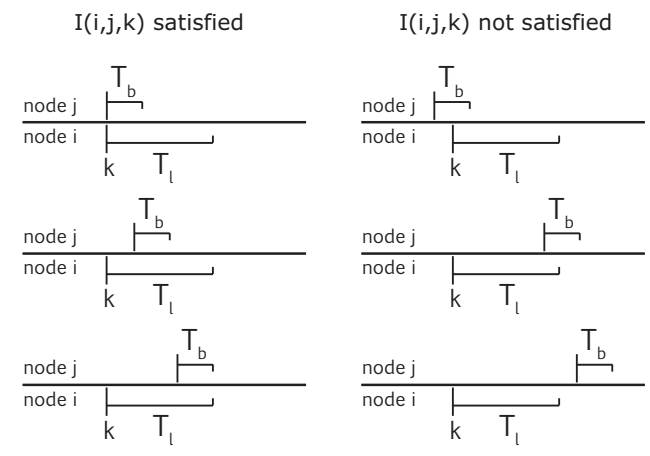

Figure 1: Examples showing when condition $I(i, j, k)$ is satisfied and when not
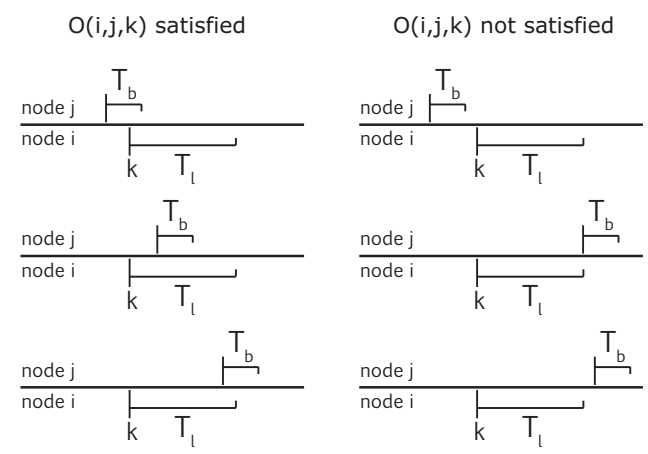

Figure 2: Examples showing when condition $O(i, j, k)$ is satisfied and when not

To determine the probability that condition $L(i, k)$ is satisfied, we first note that the probability that event $k$ of node $i$ is a listening event equals $p_{l}$. Furthermore, the probability that no other (broadcast or listening) events of its own occur in the node's listening interval is determined as $e^{-\lambda T_{l}}$, because the events are Poisson distributed with rate $\lambda$. Since these probabilities are independent, we then obtain:

$$
\operatorname{Pr}[L(i, k)]=p_{l} \cdot e^{-\lambda T_{l}}
$$

In order for condition $I(i, j, k)$ to be satisfied, one broadcasting event from node $j$ should fall inside a listening interval of node $i$ so that a whole broadcast fits within the listening interval. This means that the broadcast event from node $j$ should be in the first part with length $T_{l}-T_{b}$ of node $i$ 's listening interval and no other events from node $j$ should occur in the remaining part of the listening interval, which has total length $T_{l}$; these occurrences are statistically independent, so

$$
\operatorname{Pr}[I(i, j, k)]=
$$

$\operatorname{Pr}\left[1\right.$ broadcast event in interval of length $\left.T_{l}-T_{b}\right]$.

$$
\operatorname{Pr}\left[0 \text { broadcast events in interval of length } T_{b}\right]
$$

Since the broadcasts of node $j$ are Poisson distributed with rate $p_{b} \lambda$, we then obtain

$$
\begin{aligned}
\operatorname{Pr}[I(i, j, k)] & =p_{b} \lambda\left(T_{l}-T_{b}\right) e^{-p_{b} \lambda\left(T_{l}-T_{b}\right)} e^{-p_{b} \lambda T_{b}} \\
& =p_{b} \lambda\left(T_{l}-T_{b}\right) e^{-p_{b} \lambda T_{l}}
\end{aligned}
$$

Condition $O(i, j, k)$ is satisfied if one or more broadcasting events from node $j$ falls inside a listening interval of node $i$ or in the interval of length $T_{b}$ directly preceding this listening interval. The probability of $O(i, j, k)$ holding true then equals one minus the probability that no broadcast events from node $j$ fall in node $i$ 's listening interval of length $T_{b}+T_{l}$. Using the Poisson distribution with rate $p_{b} \lambda$ of broadcasts from node $j$, we obtain

$$
\operatorname{Pr}[O(i, j, k)]=1-e^{-p_{b} \lambda\left(T_{l}+T_{b}\right)}
$$

The probability that condition $C(i, j, k)$ is satisfied then becomes:

$$
\begin{aligned}
& \operatorname{Pr}[C(i, j, k)]= \\
& \quad=\operatorname{Pr}[L(i, k)] \operatorname{Pr}[I(i, j, k)] \prod_{r \in N_{i} \backslash\{j\}}(1-\operatorname{Pr}[O(i, r, k)]) \\
& =p_{b} p_{l}\left(T_{l}-T_{b}\right) e^{-\left(1+p_{b}\right) \lambda T_{l}} \prod_{r \in N_{i} \backslash\{j\}} e^{-p_{b} \lambda\left(T_{l}+T_{b}\right)} \\
& =p_{b} p_{l}\left(T_{l}-T_{b}\right) e^{-\left(1+p_{b}\right) \lambda T_{l}} e^{-p_{b} \lambda\left(T_{l}+T_{b}\right)\left(\left|N_{i}\right|-1\right)}
\end{aligned}
$$

In analogy to the synchronous case, this results in the matrix form $\mathbf{m}(k+1)=S \mathbf{m}(k)$ in Eq. (2), whereby matrix $S$ has as its elements:

$$
s_{i i}=1-\frac{\alpha}{\omega_{i}}\left|N_{i}\right| p_{b} p_{l} \lambda\left(T_{l}-T_{b}\right) e^{-\left(1+p_{b}\right) \lambda T_{l}} e^{-p_{b} \lambda\left(T_{l}+T_{b}\right)\left(\left|N_{i}\right|-1\right)}
$$

in the diagonal,

$$
s_{i j}=\frac{\alpha}{\omega_{i}} p_{b} p_{l} \lambda\left(T_{l}-T_{b}\right) e^{-\left(1+p_{b}\right) \lambda T_{l}} e^{-p_{b} \lambda\left(T_{l}+T_{b}\right)\left(\left|N_{i}\right|-1\right)}
$$

outside the diagonal $(i \neq j)$ for $j \in N_{i}$, and

$$
s_{i j}=0
$$

otherwise. The expression for $\mathbf{1}^{T} s_{\bullet i}$ then becomes:

$$
\begin{aligned}
& 1-\alpha p_{b} p_{l} \lambda\left(T_{l}-T_{b}\right) e^{-\left(1+p_{b}\right) \lambda T_{l}} . \\
& \quad \sum_{j \in N_{i}}\left[\frac{e^{-p_{b} \lambda\left(T_{l}+T_{b}\right)\left(\left|N_{i}\right|-1\right)}}{\omega_{i}}-\frac{e^{-p_{b} \lambda\left(T_{l}+T_{b}\right)\left(\left|N_{j}\right|-1\right)}}{\omega_{j}}\right]
\end{aligned}
$$

We then need to find values of $\omega_{i}$ and $\omega_{j}$ such that for all pairs $i$ and $j$ the following holds

$$
\frac{\omega_{j}}{\omega_{i}}=\frac{e^{-p_{b} \lambda\left(T_{l}+T_{b}\right)\left(\left|N_{j}\right|-1\right)}}{e^{-p_{b} \lambda\left(T_{l}+T_{b}\right)\left(\left|N_{i}\right|-1\right)}}
$$

In analogy to the synchronous case, we then derive the following form for $\omega_{i}$ :

$$
\omega_{i}=c e^{-p_{b} \lambda\left(T_{l}+T_{b}\right)\left(\left|N_{i}\right|-1\right)}
$$

with $c$ being a constant that does not depend on the node. The quantity

$$
e^{-p_{b} \lambda\left(T_{l}+T_{b}\right)\left|N_{i}\right|}
$$

equals the probability that all nodes in the neighborhood of node $i$ are silent during a listening interval of node $i$. This means that the same algorithm (4) as in the synchronous case can be used to estimate $\omega_{i}$.

Applying Theorem 2 in [10] to the system in Eq. (2) in the asynchronous case with balanced $\omega$ 's, we conclude that the system converges to average consensus, provided that the following condition is satisfied.

$$
\begin{aligned}
0<\alpha<\min _{i=1 \ldots n} & \omega_{i} \\
& \frac{\left|N_{i}\right| p_{l} p_{b} \lambda\left(T_{l}-T_{b}\right) e^{-\left(1+p_{b}\right) \lambda T_{l}} e^{-p_{b} \lambda\left(T_{l}+T_{b}\right)\left(\left|N_{i}\right|-1\right)}}{}
\end{aligned}
$$


Substituting the $\omega$ 's in Eq. (6) to balance for unequal node degrees gives us the following condition:

$$
0<\alpha<\min _{i=1 \ldots n} \frac{c}{\left|N_{i}\right| p_{l} p_{b} \lambda\left(T_{l}-T_{b}\right) e^{-\left(1+p_{b}\right) \lambda T_{l}}}
$$

whereby $c$ is a constant determined such that the $\omega$ 's are all within a range that optimizes convergence speeds, while guaranteeing stable dynamics of Eq. (1).

Like in the synchronous case the convergence speed becomes faster for smaller values of the largest eigenvalue $\mu_{2}$ of $S$, and smaller values of $\mu_{2}$ tend to follow from smaller values of the non-zero off-diagonal elements of matrix $S$. Substituting expression (6) for $\omega_{i}$ into $s_{i j}$, we obtain

$$
s_{i j}=\alpha p_{l} p_{b} \lambda\left(T_{l}-T_{b}\right) e^{-\left(1+p_{b}\right) \lambda T_{l}} / c
$$

for $i \neq j$. Eq. (7) then implies

$$
s_{i j}<\frac{1}{\max _{r}\left|N_{r}\right|}
$$

for $i \neq j$, which is similar to the synchronous case.

\section{NUMERICAL EVALUATIONS}

In this section we describe computer simulations of the asynchronously timed system. Numerical evaluations on a system resembling our synchronous model have been reported in [6]. We have evaluated two scenarios.

1. $\omega_{i}$ set to the constant value 1 . In this case the values of $\omega_{i}$ are not balanced, and in theory the update algorithm converges to consensus among the node, but the consensus value is not necessarily the average of all node values [10].

2. $\omega_{i}$ set to the value in Eq. (6), with the constant $c$ being defined as $c=\max _{i} e^{p_{b} \lambda\left(T_{l}+T_{b}\right)\left(\left|N_{i}\right|-1\right)}$. In this case the update algorithm converges to average consensus in theory [10].

As underlying graph we used random geometric graphs with 100 nodes. The nodes are uniform randomly distributed by $\mathrm{x}$ - and $\mathrm{y}$-coordinate in a unit area. The nodes of such graphs tend to vary in degree, and to cope with this variation we need to select optimal values for $\omega_{i}$ in case 2 . It turns out that, depending on the parameter settings, the $\omega$ 's for different nodes may vary by a factor of 100 to 1000 for values of $p_{b}$ around 0.7 . This suggests that even though convergence in the second case may be closer to the actual average, it is likely to be slower than that in the first case, because some nodes will converge much slower than others. To test this proposition, we conduct simulations with the following parameter settings. The rate of the Poisson process is set to $\lambda=1 / 20$, which means that on average every 20 time units one Poisson event will occur for a node. The intervals for listening and broadcasting are set to $T_{l}=10$ and $T_{b}=2$ time units, respectively, and the corresponding probabilities have the values $p_{l}=0.8$ and $p_{b}=1-p_{l}=0.2$. The value of $\alpha$ is varied from 0.1 to 0.5 with step size 0.1 , and for each parameter setting the simulation is repeated 100 times. The squared error $(\bar{x}(t)-\bar{x}(0))^{2}$ is averaged over those simulations, with $\bar{x}(t)$ and $\bar{x}(0)$ denoting the averages over all node values at times $t$ and 0 , respectively. Furthermore, we keep track of the number of time units required to reach convergence to a stage in which the standard deviation of all node values do not exceed 0.1 .
The results of the simulations are given in Figs. 3 and 4 with the error bars indicating confidence intervals of $95 \%$.

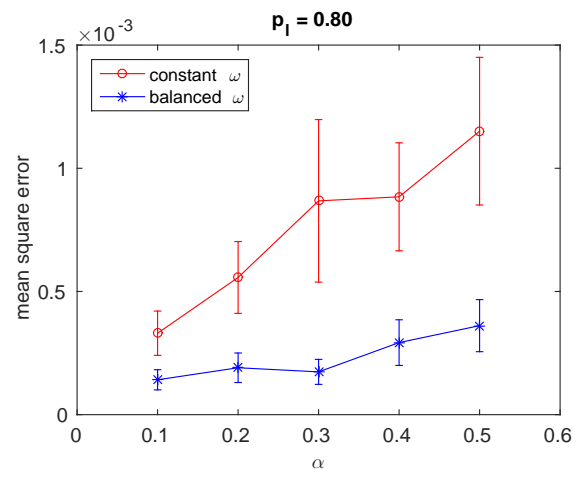

Figure 3: Squared errors for the case $\omega_{i}$ is constant 1 respectively balanced, with $p_{l}=0.8$, and $\alpha$ varying from 0.1 to 0.5 in step sizes of 0.1 . Results are averaged over 100 simulations, and error bars indicate 95\% confidence intervals.

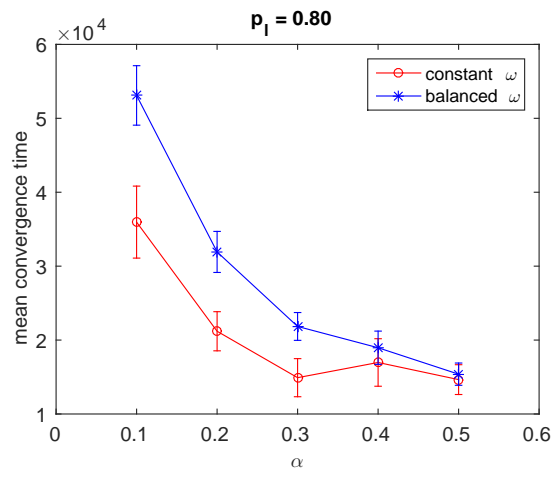

Figure 4: Convergence times for the case $\omega_{i}$ is constant 1 respectively balanced, with $p_{l}=0.8$, and $\alpha$ varying from 0.1 to 0.5 in step sizes of 0.1 . Results are averaged over 100 simulations, and error bars indicate $95 \%$ confidence intervals. Since the Poisson rate is $\lambda=1 / 20$, the number of Poisson events for each node to convergence is $1 / 20$ th of the convergence time. Note that not each Poisson event results in an update of a node's value, since - depending on the parameter settings - broadcasts may drop out or collide.

Convergence for balanced $\omega$ 's is indeed more precise than that for constant $\omega$ 's, and convergence speed tends to be slower in the former case. However, for the largest value of $\alpha$ we experimented with, i.e., $\alpha=0.5$, there is virtually no difference in speed. Though the squared errors for the model with constant $\omega$ 's are larger than for the balanced $\omega$ 's, the difference is marginal, so the use of constant $\omega$ 's can be justified for parameter settings that have significant differences in convergence speed.

To obtain a better impression of the behavior of the asynchronous consensus algorithm for constant $\omega$ settings, we conduct simulations over a wider range of parameter values. In particular, we vary both $\alpha$ and $p_{l}$ from 0.1 to 0.9 in steps of 0.1 , and again average our results over 100 simulations. 
The squared error, shown in Fig. 5, tends to decrease for smaller values of $\alpha$, but it is less sensitive to the value of $p_{l}$.

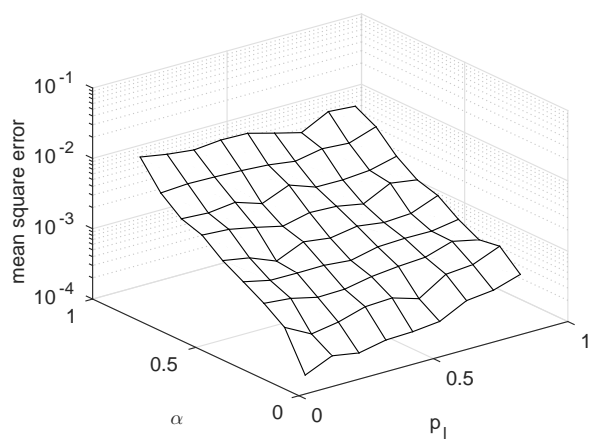

Figure 5: Mean squared errors averaged over 100 simulations when $\omega_{i}$ is constant and the values of $\alpha$ and $p_{l}$ both vary from 0.1 to 0.9 in steps of 0.1 .

The convergence time, shown in Fig. 6, appears to be optimal at a point near $\alpha=0.4$ and $p_{l}=0.2$.

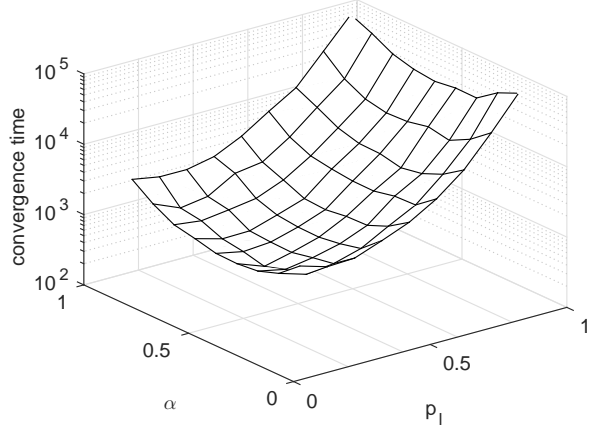

Figure 6: Convergence times averaged over 100 simulations when $\omega_{i}$ is constant and the values of $\alpha$ and $p_{l}$ both vary from 0.1 to 0.9 in steps of 0.1 . The minimal achieved convergence time is for $\alpha=0.4$ and $p_{l}=0.2$.

\section{CONCLUSIONS}

The asynchronous average consensus algorithm proposed in this paper is suitable for wireless sensor networks in which nodes are available in high densities, because communication is localized and the consensus value reached after convergence needs to be read out by a server from only one node; this significantly reduces wireless network traffic. The nodes communicate by broadcasting their values without expecting any acknowledgments from neighboring nodes, which contributes to an extremely simple communication protocol. Though this paper focuses on average consensus, the mechanisms underlying the algorithm can be readily employed in other consensus type algorithms.

The proposed algorithm is employed on random geometric graphs, which are known to give slow convergence for consensus algorithms due to their two-dimensional geometric character. The convergence speed tend to be worse for the model with balanced $\omega$ 's as compared to the model with constant $\omega$ 's due to the large differences in the values of the $\omega$ 's in the balanced case. The squared errors do differ between both cases, but they are small enough that a model with constant $\omega$ 's can be justified. Though this model does not necessarily converge to average consensus in theory, in practice it does. We speculate that the randomness of the underlying graphs play a role in evening out differences at which update values propagate along different areas of the graph. Future work will include simulations to verify the validity of this hypothesis.

\section{ACKNOWLEDGMENTS}

This work was supported by JSPS KAKENHI Grant Number JP16H01719.

\section{REFERENCES}

[1] S. Boyd, A. Ghosh, B. Prabhakar, and D. Shah. Randomized gossip algorithms. IEEE/ACM Transactions on Networking, 14(SI):2508-2530, Jun 2006.

[2] J. Dall and M. Christensen. Random geometric graphs. Physical Review E, 66:016121, July 2002.

[3] J. Diaz, D. Mitsche, and X. Perez-Gimenez. Large connectivity for dynamic random geometric graphs. IEEE Transactions on Mobile Computing, 8(6):821-835, June 2009.

[4] A. Dimakis, A. Sarwate, and M. Wainwright. Geographic gossip: Efficient averaging for sensor networks. IEEE Transactions on Signal Processing, 56(3):1205-1216, Mar 2008.

[5] A. G. Dimakis, S. Kar, J. M. F. Moura, M. G. Rabbat, and A. Scaglione. Gossip algorithms for distributed signal processing. Proceedings of the IEEE, 98(11):1847-1864, Nov 2010.

[6] P. Frasca and J. M. Hendrickx. Large network consensus is robust to packet losses and interferences. In European Control Conference (ECC), pages 1782-1787, July 2013.

[7] P. Gupta and P. Kumar. The capacity of wireless networks. IEEE Transactions on Information Theory, 46(2):388-404, March 2000.

[8] J. Macaulay, L. Buckalew, and G. Chung. The internet of things in logistics. Technical report, DHL and Cisco, Trend Report, 2015.

[9] S. Maghsudi and E. Hossain. Distributed cell association for energy harvesting iot devices in dense small cell networks: A mean-field multi-armed bandit approach. arXiv:1605.0005\%v1, April 2016.

[10] R. Olfati-Saber, J. Fax, and R. Murray. Consensus and cooperation in networked multi-agent systems. Proceedings of the IEEE, 95(1):215-233, Jan 2007.

[11] M. Penrose. Random Geometric Graphs. Oxford University Press, 2003.

[12] L. Xiao and S. Boyd. Fast linear iterations for distributed averaging. Systems \& Control Letters, 53(1):65 - 78, 2004.

[13] L. Xiao, S. Boyd, and S.-J. Kim. Distributed average consensus with least-mean-square deviation. Journal of Parallel and Distributed Computing, 67(1):33-46, Jan 2007. 